\title{
Splitting phenomenon of a higher-order shallow water theory associated with a longitudinal planetary waves
}

\author{
Ranis N. Ibragimov ${ }^{1,2}$, Guang, Lin ${ }^{2}$ \\ ${ }^{1}$ Applied Statistics Lab \\ GE Global Research \\ 1 Research Circle \\ Niskayuna, NY 12309 \\ ${ }^{2}$ Computational Mathematics Group, \\ Pacific Nothwest National Laboratory \\ 902 Battelle Boulevard \\ Richland, WA 99352, USA
}

September 11, 2014

\begin{abstract}
The Cauchy-Poisson free boundary problem associated with a nonstationary motion of a perfect incompressible fluid circulating around the equatorial plane of a planet is considered. It is shown that the corresponding theory of a higher-order shallow approximation admits two functionally independent systems, while the classical problem for the flat bottom admits only one system.
\end{abstract}

\section{Introduction}

The large-scale atmospheric dynamics is usually described by moving air masses on a sphere or circle in terms of three and two dimensional Navier-Stokes or Euler equations a thin rotating spherical shell ([6], [7]; [28], [29]; [15], [16], [19], [22], [25], [26], [18]) or within the theory of shallow water approximation ([27]; [41], [21], [24], [30]). The modeling of such moving air masses plays an important role in understanding the global climate control ([39]; [1]; [8]). The inclusion of a spherical shape on global scales creates a cyclonic rotation around the poles, i.e., west-to-east winds ([3]; [19]). Namely, as has been indicated already in the late 1800s by Herrmann in [13], the temperature difference between the equator and the poles of a sphere gives rise to waves of two kinds. The first kind consists of waves that advance in the direction of the meridian; the second kind includes equatorial waves. The atmospheric pressures and motions resulting from the combination of these two groups of intersecting waves give rise to the cyclonic and anticyclonic phenomena which are nowadays a paramount topic of research in atmospheric modeling.

Permanent water waves have been considered in a large number of papers. However, most researchers are concerned with fluid motion which is infinitely deep and extends infinitely both rightward and leftward (see e.g. [9]; [32] or [38] for the history). Such problems are usually 


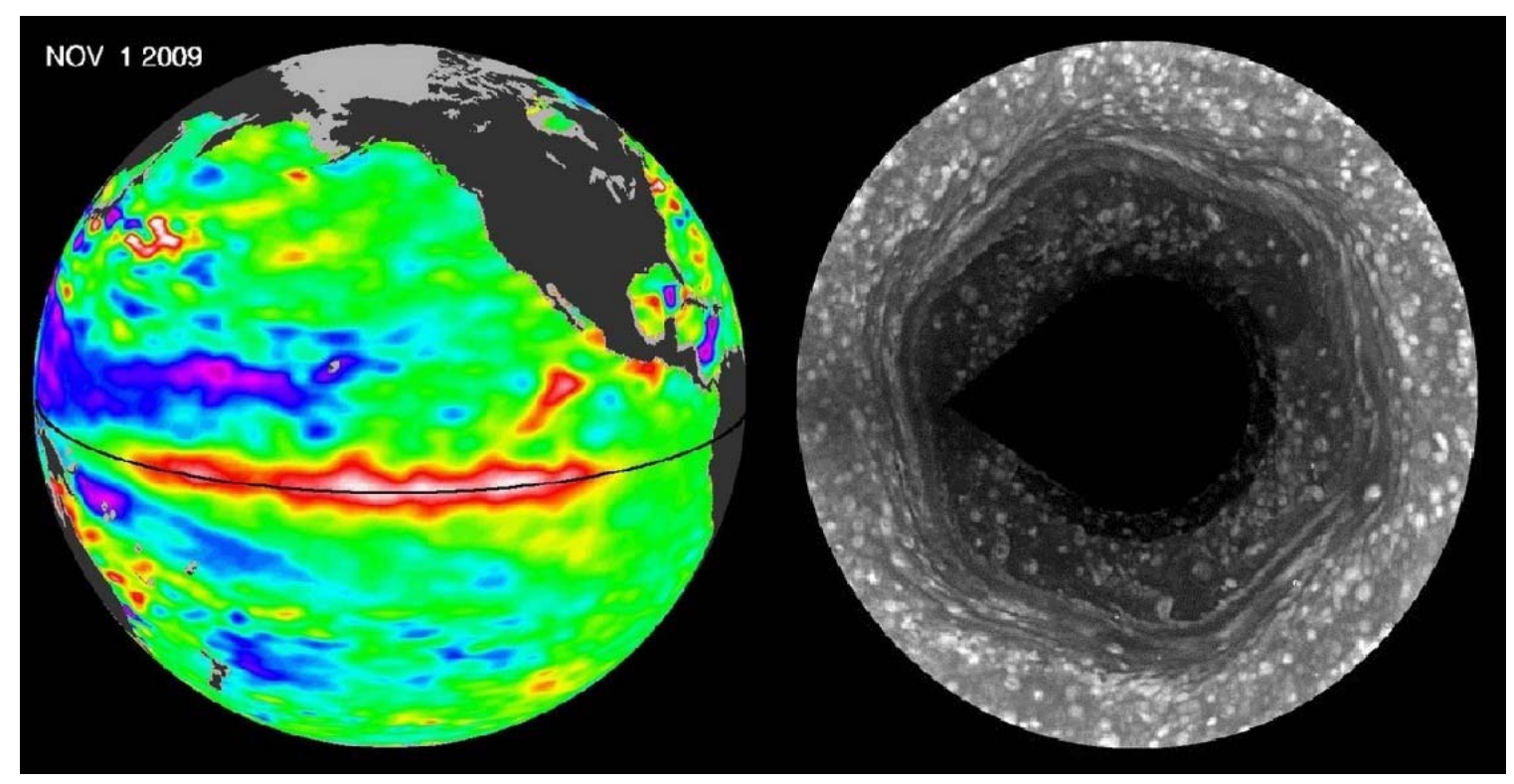

Figure 1: Left: Sea-level height data from November 2009 showing the dynamics of warm water known as Kelvin waves that can be seen traveling eastward along the equator (black line) in Nov. 01, 2009 image. El Ninos form when trade winds in the equatorial western Pacific relax over a period of months, sending Kelvin waves eastward across the Pacific like a conveyor belt. Image credit: NASA/JPL. Right: Image from Cassini, made possible only as Saturn's north pole emerged from winter darkness, shows new details of a jet stream that follows a hexagon-shaped path and has long puzzled scientists.

called Stokes's problem if the surface tension is neglected and Wilton's problem if the surface tension is taken into account (see e.g. [30]). In the first approximation, shallow water equations represent the mathematical theory that can be used to investigate the fluid flows in channels (see e.g. [11]). However, this theory does not reveal the role the role of an undisturbed level of the fluid surface which is needed to determine the precision of the first approximation. A higher order approximation is derived in this work. Here we derive a shallow water equations modeling a simple longitudinal atmospheric motion. For the sake of simplicity, the motion of the fluid is supposed to be irrotational and pressure on a free boundary is constant. It is postulated that the fluid depth is small compared to the radius of the circle and the gravity vector is directed to the center of the circle. As has been discussed in [24] and in [20], under these assumptions, this problem can be associated with an atmospheric longitudinal circulation around equatorial plane.

Such model can be associated e.g. with a large-scale eastward moving, wave of warm water, known as a Kelvin wave that can be seen traveling eastward along the equator as shown in the left Panel of Figure 1. Particularly, in the central and eastern equatorial Pacific, the warm wave appears as the large area of higher-than-normal sea surface heights - warmer-than-normal sea surface temperatures - between 170 degrees east and 100 degrees west longitude.

Another spectacular example of circulating waves is domesticated on the left panel of Figure 1 showing a jet stream that follows a hexagon-shaped path at the north pole of Saturn. The hexagon was originally discovered in images taken by Voyager spacecraft in the early 1980s. 
This image also shows another unexplained phenomena such as waves that can be seen traveling along hexagon. These waves and the six-sided shape of the jet stream remain a mystery up to the date.

In earlier work, [19] and [16] the exact solutions associated with an incompressible viscous and non-viscous fluid flows in a thin spherical shell were found and investigated. Here we aim to investigate the shallow water model associated with long waves formed in the equatorial plane. It is shown that at the higher order approximation the splitting phenomenon of shallow water theory holds, i.e. the model admits two different systems of shallow water equations, which was not observed for a classical problem for the flat bottom. Additionally, the singular exact solutions are provided for the exact mathematical model describing longitudinal planetary waves. Computational experiments [27]; [5]; [40] and [41] provide a credible evidence to support the assertion that singular solutions to the shallow water equations may exist on a stationary sphere. They also suggest that singular solutions are less likely on a rotating sphere. However, the experiments conducted up to the date are not sufficiently extensive to support any credible evidence on the existence or nonexistence of singular solutions to the shallow water equations on a rotating sphere. From the practical standpoint, it is useful to note that the fluid particles at the North and South Poles spin around themselves at a rate $\Omega=2 \pi \mathrm{rad} /$ day, whereas fluid particles in the polar (latitude) domain $\theta \in\left[\theta_{0}, \pi-\theta_{0}\right]$ do not spin around themselves but simply translate provided $\theta_{0} \in\left(0, \frac{\pi}{2}\right)$. Thus the achievable meteorological flows rotating around the poles correspond to the flows that are being translated along the equatorial plane.

\section{The model}

We introduce polar coordinates $x=r \cos \theta, y=r \sin \theta$ and use the following notation: $R$ is the radius of the Earth, $\theta$ is a polar angle, $r$ is the distance from the origin, $h=h_{0}+\eta(t, \theta)$, where $h_{0}$ is undisturbed level of atmosphere above the Earth and $\eta(t, \theta)$ is the level of disturbance of a free boundary, as shown schematically in Figure 2. It is supposed in what follows that $\theta \in[0,2 \pi]$ while $r \in[R, h(t, \theta)]$. The homogeneous gravity field $\vec{g}$ is assumed to be a constant and directed to the center of the Earth. The restriction $\theta \in[0,2 \pi]$ appears for the following reason: the velocity potential $\varphi(\varsigma)$ can be introduced by the analyticity of the complex potential $\vartheta(\varsigma)=\varphi+i \psi$, where $\varsigma=r e^{i \theta}$ is the independent complex variable and $\psi(\varsigma)$ is the stream function. Correspondingly, the complex velocity $d \vartheta / d \varsigma$ is a single-valued analytic function of $\varsigma$, although $\vartheta$ is not single-valued. In fact, when we turn around the bottom $r=R$ once,

$\varphi$ increases by $-\int_{0}^{2 \pi} \frac{\partial \psi}{\partial r}(R, \theta) d \theta$ which has a positive sign by the maximum principle (Hopf's lemma). Hence, if we remove the width of annulus region $\theta=0, r \in\left[R, R+h_{0}\right]$, then at every point $(r, \theta)$, the complex potential $\vartheta(\varsigma)$ is a single-valued analytic function.

We start with the usual assumption that the velocity field $\vec{v}=\left(v_{r}, v_{\theta}\right)$ satisfies the Euler's equations and the no-leak condition $v_{r}=0$ on a solid bottom $r=R$. We also assume the kinematic condition on the free boundary. Namely, the velocity on the free boundary $r=$ $R+h(t, \theta)$ is tangential to the free boundary. We define the free boundary by equation $f=r-h(\theta, t)=0$ so that the kinematic condition is written as

$$
\frac{d f}{d t}=\frac{\partial f}{\partial t}+\vec{v} \nabla f=0
$$




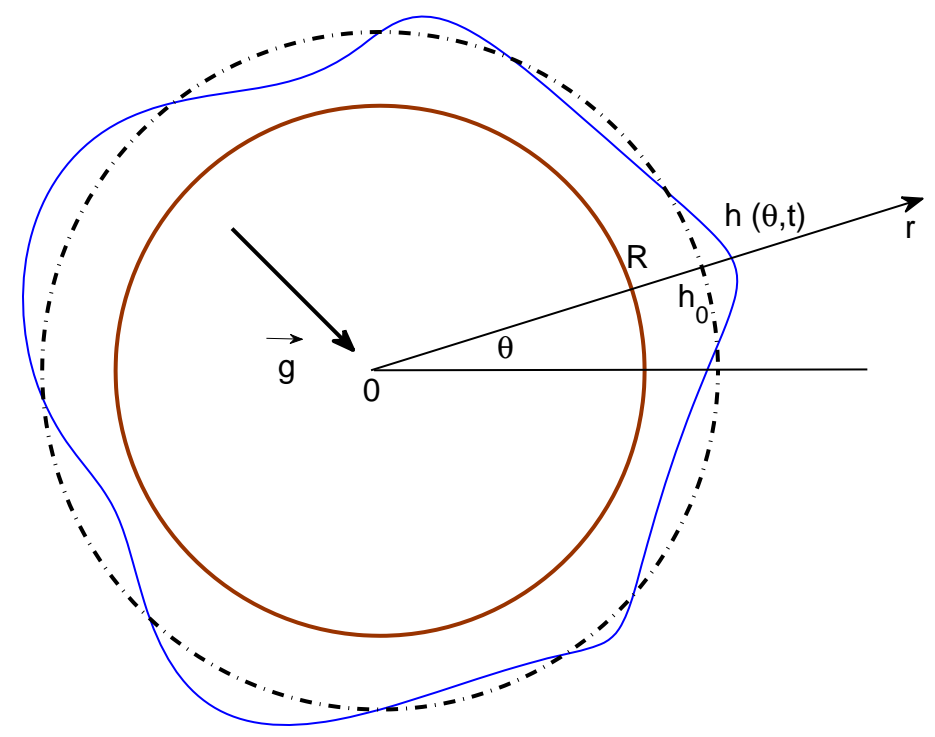

Figure 2: Schematic showing a longitudinal atmospheric motion circulating around the Earth.

where

$$
\nabla=\left(\frac{\partial}{\partial r}, \frac{1}{r} \frac{\partial}{\partial \theta}\right)
$$

In what follows, it is assumed that the fluid motion is potential in the domain of the motion which allows to introduce the stream function $\psi(t, r, \theta)$ via

$$
v_{r}=-\frac{1}{r} \frac{\partial \psi}{\partial \theta}, \quad v_{\theta}=\frac{\partial \psi}{\partial r}
$$

So that the no-leak condition on the solid boundary can be written as $\psi(R, \theta, t)=0$ whereas the kinematic condition (1) takes the form

$$
\frac{\partial h}{\partial t}+\frac{1}{r} \frac{\partial \psi}{\partial \theta}+\frac{1}{r} \frac{\partial \psi}{\partial r} \frac{\partial h}{\partial \theta}=0
$$

Since

$$
v_{r}=-\frac{1}{r} \int_{R}^{R+h} \frac{\partial v_{\theta}}{\partial \theta} d r
$$

we can also write Eq. (4) at the free boundary $r=R+h$ as the mass balance equation. i.e.,

$$
\frac{\partial h}{\partial t}+\frac{1}{R+h} \frac{\partial}{\partial \theta} \int_{R}^{R+h} v_{\theta} d r=0
$$


We next define the average velocity $u(\theta, t)$ as

$$
u(\theta, t)=\frac{1}{h} \int_{R}^{R+h} v_{\theta}(r, \theta, t) d r=\frac{1}{h} \psi(R+h, \theta, t) .
$$

In terms of the average velocity, the kinematic condition (4) is written as

$$
\frac{\partial h}{\partial t}+\frac{1}{r} \int_{R}^{R+h} \frac{\partial v_{\theta}}{\partial \theta} d r+\frac{1}{r} \frac{\partial \psi}{\partial r} \frac{\partial h}{\partial \theta}=0 .
$$

Finally, the dynamic condition is obtained from the requirement that the pressure $p$ is constant at the free boundary $r=R+h(\theta, t)$. Thus, projecting of the impulse equation

$$
\frac{\partial \vec{v}}{\partial t}+\nabla\left(\frac{1}{2}|\vec{v}|^{2}\right)+\frac{1}{\rho} \nabla p=\vec{g}
$$

on the tangential vector

$$
\vec{\tau}=\left(\frac{1}{r} \frac{\partial h}{\partial \theta}, 1\right)
$$

to the free boundary yields

$$
\frac{1}{r} \frac{\partial h}{\partial \theta} \frac{\partial v_{r}}{\partial t}+\frac{\partial v_{\theta}}{\partial t}+\frac{1}{r} \frac{\partial h}{\partial \theta} \frac{\partial}{\partial r} \frac{1}{2}\left(v_{r}^{2}+v_{\theta}^{2}\right)+\frac{1}{r} \frac{\partial}{\partial \theta} \frac{1}{2}\left(v_{r}^{2}+v_{\theta}^{2}\right)+\frac{1}{\rho}\left(\frac{1}{r} \frac{\partial h}{\partial \theta} \frac{\partial p}{\partial r}+\frac{1}{r} \frac{\partial p}{\partial \theta}\right)=0,
$$

where $\rho$ is a constant fluid density.

Thus, since $\left.p\right|_{r=R+h}=$ const. and $\psi$ is the harmonic function at the domain of the fluid motion, the model describing a longitudinal atmospheric motion around the Earth can be written as the following free boundary problem:

$$
\begin{gathered}
\frac{\partial^{2} \psi}{\partial \theta^{2}}+r^{2} \frac{\partial^{2} \psi}{\partial r^{2}}+r \frac{\partial \psi}{\partial r}=0 \quad(R<r<R+h), \\
\psi(R, \theta, t)=0, \\
\psi(R+h, \theta, t)=u(\theta, t) h, \\
\frac{\partial^{2} \psi}{\partial t \partial r}-\frac{1}{r^{2}} \frac{\partial h}{\partial \theta} \frac{\partial^{2} \psi}{\partial t \partial \theta}+\frac{1}{2 r} \frac{\partial}{\partial \theta}\left[\frac{1}{r^{2}}\left(\frac{\partial \psi}{\partial \theta}\right)^{2}+\left(\frac{\partial \psi}{\partial r}\right)^{2}\right]+\frac{g}{r} \frac{\partial h}{\partial \theta}=0, \quad(r=R+h), \\
r \frac{\partial h}{\partial t}+\frac{\partial}{\partial \theta}(u h)=0, \quad(r=R+h) .
\end{gathered}
$$

One can check by direct differentiation that there exists an exact stationary solution to the model (10)-(14) given by

$$
\eta=0, \quad \psi=-\frac{\Gamma}{2 \pi} \log \left(\frac{r}{R}\right)
$$




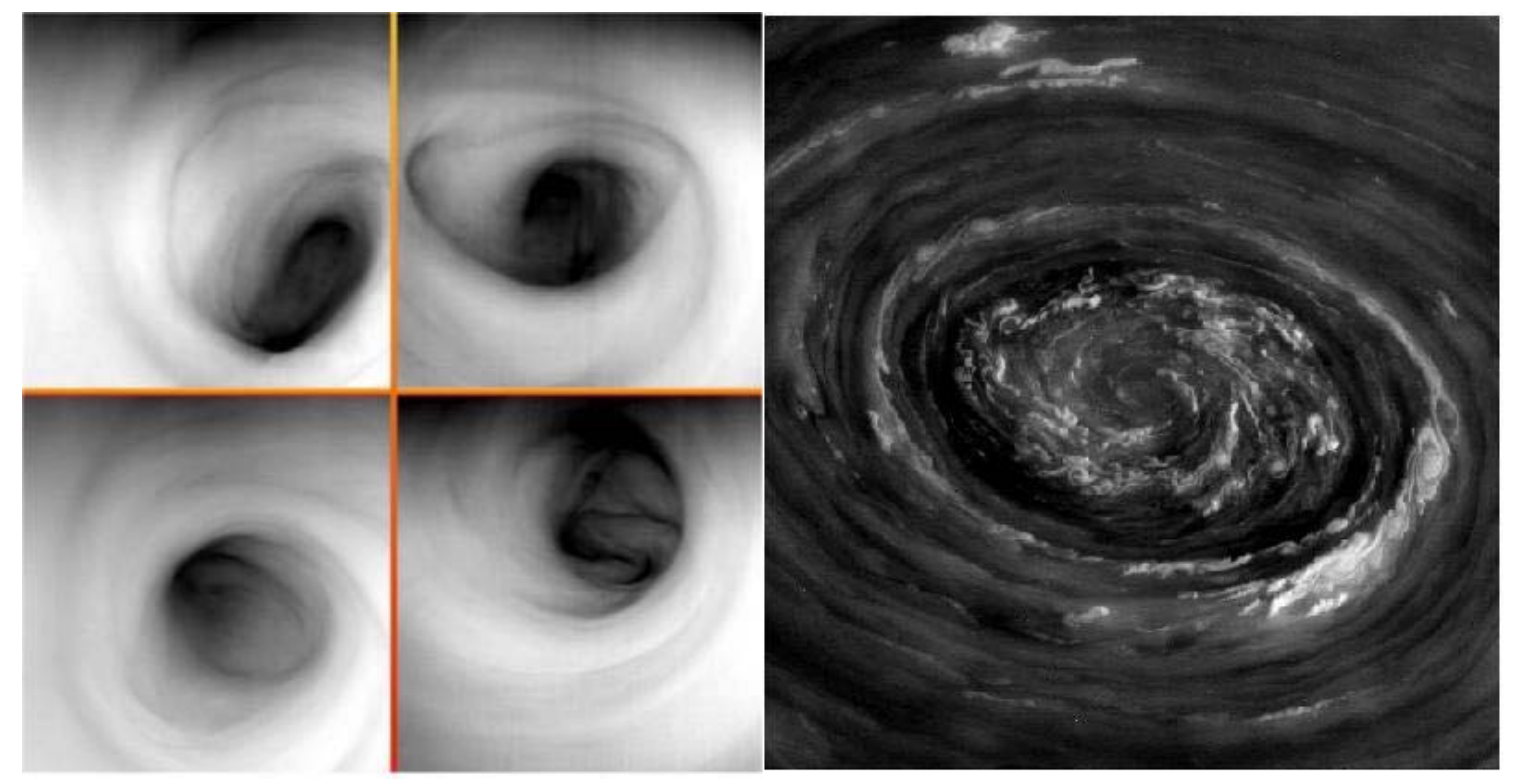

Figure 3: Left: Recent pictures of a polar vortex on Venus which is attributed to cloud formations on the palnet leaving an unexplained dark hole. It has been iscovered at Venus' north pole by the Pioneer Venus spacecraft in 1979. Credit: ESA/Virtis/INAF-IASF/Obs. de Paris-LESIA Right: Picture taken by the Cassini spacecraft of clouds circling over Saturn's north pole.

where $\Gamma=$ const.is intensity of the vortex (source) localized at the center of the earth and is related with the the rotation rate of the earth (angular velocity $\Omega=2 \pi \mathrm{rad} /$ day $\approx 0.73 \times 10^{-4}$ $\mathrm{s}^{-1}$ ) by the equation $\Gamma=2 \pi \Omega R^{2}$. The solution (15) corresponds to the constant flow with an undisturbed free surface. Thus, since the vortex is isolated ( $R$ is a solid boundary), this exact solution represent a flow whose streamlines can be visualized as flow whose streamlines are concentric circles with the common center at the origin.

At certain extent, the above ansatz (15) can also be associated with such atmospheric phenomena as illustrated in Figure 3 which is used to show the NASA images of a polar vortex on Venus (Left panel) and clouds circling over Saturn's north pole (Right panel).

Particularly, it is believed that the polar vortex shown in the left panel of Figure 3 is a very powerful whirlpool swirling steadily around the planet's poles at all times. It might be caused by a gigantic hurricane with two calm, dark eyes. This double-eyed feature, dubbed the "dipole of Venus," was thought to form when warm air from the planet's equator rose and traveled toward the pole, where it cooled and sank to form a deep, swirling atmospheric pit. For decades, astronomers expected to find a similar vortex at Venus' south pole. While Venus itself rotates slowly, just once every 117 Earth days, its atmosphere whips around the planet once every four Earth days. This "super-rotating" atmosphere ought to form massive storms at both poles, astronomers reasoned [2]; [31]. The image of the clouds circling over Saturn's north pole shown in the right panel of Figure 3 are taken by the Cassini spacecraft from a distance of about 380,000 kilometers and represents the stunning detail in Saturn's atmosphere. Clouds rise and sink and get stretched out, forming long valleys and ridges, streamers circling the planet's pole. This vortex is over 2000 kilometers; that's far bigger than a fully mature hurricane on Earth, 
but unlike a terrestrial cyclone, this may be a permanent feature in Saturn's atmosphere (see also [4]).

\section{Shallow water approximation}

It is useful to recast the model in nondimensional form by introducing the following dimensionless variables:

$$
\begin{aligned}
\theta & =\widehat{\theta}, \quad r=R+h_{0} \widehat{r}, \quad h=h_{0} \widehat{h}, \quad t=\frac{R \widehat{t}}{\sqrt{g h_{0}}}, \\
\psi & =h_{0} \sqrt{g h_{0}} \widehat{\psi}, \quad u=\sqrt{g h_{0}} \widehat{u} .
\end{aligned}
$$

We next introduce the parameter

$$
\varepsilon=\frac{h_{0}}{R}
$$

Of course, water is shallow if the parameter $\varepsilon$ is small. So, in the present model (10) - (14), the functions $\eta(\theta, t)$ and $\psi(r, \theta, t)$ are two unknown functions whereas the parameter $\varepsilon$ is a given parameter. Although shallow water theory is usually related to the case when the water depth is small relative to the wavelengths of the waves, we find it more appropriate to choose the radius of the earth $R$ as a natural physical scale since, in the frame of the present model, we consider waves with wavelengths of the order of the radius of a planet. It is considered that the flow does not depend on depth. This is exactly true for barotropic non-frictional motions. Turbulent viscosity introduces vertical current shear. However, vertically integrated volume transports are affected by the surface and bottom stress only (see e.g. [17]; [23]; [36]).

The the dynamic condition (13) is then nondimensionalized as follows:

$$
\frac{\partial^{2} \psi}{\partial t \partial r}-\frac{\varepsilon^{2}}{(1+\varepsilon h)^{2}} \frac{\partial^{2} \psi}{\partial t \partial \theta} \frac{\partial h}{\partial \theta}+\frac{1}{2(1+\varepsilon h)} \frac{\partial}{\partial \theta}\left(\frac{\varepsilon^{2}}{(1+\varepsilon h)^{2}}\left(\frac{\partial \psi}{\partial \theta}\right)^{2}+\left(\frac{\partial \psi}{\partial r}\right)^{2}\right)+\frac{1}{(1+\varepsilon h)} \frac{\partial h}{\partial \theta}=0 .
$$

Following the Lagrange's method we represent the stream function $\psi$ by the following series expansion: $\psi=\sum_{n} \varepsilon^{n} \psi^{(n)}$. Then the Laplace equation (10) takes the form

$$
\begin{gathered}
\frac{\partial^{2} \psi^{(0)}}{\partial r^{2}}+\varepsilon\left(\frac{\partial^{2} \psi^{(1)}}{\partial r^{2}}+2 r \frac{\partial^{2} \psi^{(0)}}{\partial r^{2}}+\frac{\partial \psi^{(0)}}{\partial r}\right) \\
+\varepsilon^{2}\left(\frac{\partial^{2} \psi^{(0)}}{\partial \theta^{2}}+\frac{\partial^{2} \psi^{(2)}}{\partial r^{2}}+2 r \frac{\partial^{2} \psi^{(1)}}{\partial r^{2}}+r^{2} \frac{\partial^{2} \psi^{(0)}}{\partial r^{2}}+\frac{\partial \psi^{(1)}}{\partial r}+r \frac{\partial \psi^{(0)}}{\partial r}\right)+0\left(\varepsilon^{3}\right)=0 .
\end{gathered}
$$

A comparison of the terms with the same order $\varepsilon$ in equation (18) yields a recurrent system of differential equations for the determination of all functions $\psi^{(n)}$, i.e. the Lagrange method consists in presentation of $\psi$ as the solution of the Cauchy problem with boundary conditions (11) - (12) for $\psi^{(0)}$ and zero boundary conditions for $\psi^{(1)}$ and $\psi^{(2)}$.

Thus, up to the order $\varepsilon^{2}$, the function $\psi$ is determined as follows:

$$
\psi=u r+\varepsilon\left(u \frac{r^{2}}{2}-u h \frac{r}{2}\right)+\varepsilon^{2}\left(u h \frac{r^{2}}{4}-u_{\theta \theta} \frac{r^{3}}{6}+u_{\theta \theta} h^{2} \frac{r}{6}-u h^{2} \frac{r}{4}\right)
$$


Note that the unknowns $u$ and $h$ are related by the dynamic and conditions (17) and the kinematic condition (14) which is written in nondimensional form as follows:

$$
\frac{1}{2} \frac{\partial}{\partial t}\left(\varepsilon h^{2}+2 h\right)+\frac{\partial}{\partial \theta}(u h)=0 .
$$

Using the Taylor series expansion

$$
(1+\varepsilon h)^{-1}=1-\varepsilon h+\varepsilon^{2} h^{2}+0\left(\varepsilon^{3}\right),
$$

and keeping the terms $0\left(\varepsilon^{2}\right)$, we write the dynamic condition (17) as

$$
\begin{gathered}
\frac{\partial^{2} \psi}{\partial t \partial r}+\frac{1}{2} \frac{\partial}{\partial \theta}\left(\varepsilon^{2}\left(\frac{\partial \psi}{\partial \theta}\right)^{2}+\left(\frac{\partial \psi}{\partial r}\right)^{2}\right)-\varepsilon^{2} \frac{\partial^{2} \psi}{\partial t \partial \theta} \frac{\partial h}{\partial \theta} \\
+\left(\frac{\varepsilon h}{2} \frac{\partial}{\partial \theta}\left(\frac{\partial \psi}{\partial r}\right)^{2}+\varepsilon h \frac{\partial h}{\partial \theta}\right)(\varepsilon h-1)+\frac{\partial h}{\partial \theta}=0 .
\end{gathered}
$$

We note that we can also multiply equation $(17)$ by $(1+\varepsilon h)^{2}$ first and then use the Taylor series expansion (21). In this case we obtain

$$
\begin{gathered}
\frac{\partial^{2} \psi}{\partial t \partial r}+\frac{1}{2} \frac{\partial}{\partial \theta}\left(\varepsilon^{2}\left(\frac{\partial \psi}{\partial \theta}\right)+\left(\frac{\partial \psi}{\partial r}\right)^{2}\right)-\varepsilon^{2} \frac{\partial^{2} \psi}{\partial t \partial \theta} \frac{\partial h}{\partial \theta} \\
+\left([2+\varepsilon h] \frac{\partial^{2} \psi}{\partial t \partial r}+\frac{1}{2} \frac{\partial}{\partial \theta}\left(\frac{\partial \psi}{\partial r}\right)^{2}+\frac{\partial h}{\partial \theta}\right) \varepsilon h+\frac{\partial h}{\partial \theta}=0 .
\end{gathered}
$$

\section{Illustration of splitting phenomenon for shallow water theory}

If we substitute $\psi$ given by (19) into equation (23), we get the system of shallow water equations:

$$
\begin{gathered}
F_{1}=u_{t}+u u_{\theta}+h_{\theta}+\varepsilon\left(\frac{5 h}{2} u_{t}-\frac{u}{2} h_{t}+2 h u u_{\theta}-\frac{u^{2}}{2} h_{\theta}+h h_{\theta}\right)+ \\
\varepsilon^{2}\left(\frac{9}{4} h^{2} u_{t}-\frac{h^{2}}{3} u_{\theta \theta t}+\frac{1}{3} u_{\theta \theta} h h_{t}-u_{t \theta} h h_{\theta}+h u_{\theta}+u_{\theta \theta} h+\frac{7}{4} u u_{\theta} h^{2}-\right. \\
\left.\frac{3}{4} u^{2} h h_{\theta}-\frac{h^{2}}{3} u u_{\theta \theta \theta}+\frac{1}{3} u u_{\theta \theta} h h_{\theta}-\frac{h^{2}}{3} u_{\theta} u_{\theta \theta}\right)+o\left(\varepsilon^{3}\right)=0, \\
\frac{1}{2} \frac{\partial}{\partial t}\left(\varepsilon h^{2}+2 h\right)+\frac{\partial}{\partial \theta}(u h)=0 .
\end{gathered}
$$

Now, if we substitute $\psi$ given by (19) into equation (22), we arrive at the following system of nonlinear shallow water equations:

$$
F_{2}=u_{t}+u u_{\theta}+h_{\theta}+\varepsilon\left(\frac{5 h}{2} u_{t}-\frac{u}{2} h_{t}+2 h u u_{\theta}-\frac{u^{2}}{2} h_{\theta}+h h_{\theta}-2 h\left[u_{t}+u u_{\theta}+h_{\theta}\right]\right)+
$$




$$
\begin{gathered}
\varepsilon^{2}\left(\frac{h^{2}}{4} u_{t}-\frac{h^{2}}{3} u_{\theta \theta t}+\frac{1}{3} u_{\theta \theta} h h_{t}-u_{t \theta} h h_{\theta}+\frac{2}{3} h^{2} u_{\theta} u_{\theta \theta}+\right. \\
\left.\frac{3}{4} h^{2} u u_{\theta}+\frac{1}{3} h h_{\theta} u u_{\theta \theta}-\frac{h^{2}}{3} u u_{\theta \theta \theta}+h^{2} h_{\theta}\right)+o\left(\varepsilon^{3}\right)=0 \\
\frac{1}{2} \frac{\partial}{\partial t}\left(\varepsilon h^{2}+2 h\right)+\frac{\partial}{\partial \theta}(u h)=0 .
\end{gathered}
$$

Thus the model (10) - (14) admits two systems of shallow water equations. We call it splitting phenomenon of shallow water theory. We remark that the equations (24) and (26) have the structure

$$
\begin{gathered}
F_{1}=\alpha_{1}+\varepsilon \alpha_{2}+\varepsilon^{2} \alpha_{3}, \\
F_{2}=\alpha_{1}+\varepsilon\left(\alpha_{2}-2 h \alpha_{1}\right)+\varepsilon^{2} \alpha_{4} .
\end{gathered}
$$

We next consider two particular examples that are aimed to illustrate the splitting phenomena for the higher-order shallow water theory associated with a longitudinal planetary waves.

\subsection{Example: linear non-stationary dispersion analysis}

We consider a simple form of perturbation

$$
u=u_{0}+A(\xi), \quad h=h_{0}+B(\xi), \quad|A|<<1,|B|<<1
$$

resented by a small surface-elevation amplitude with a constant wavelength, propagating with the phase velocity associated with the splitted system (24)-(25) and (26)-(27). According to the linear theory for waves forced by gravity, we will look for the solution of the form (30) in which $\xi(\theta, t)=k \theta-\omega t$ is the phase function (in radians), depending on the angular position $\theta(\theta$, in meters) and time $t(t$, in seconds). Additionally, in formula (30) we use the following notation: $k=2 \pi / \lambda$ is a wave number (in radians per metre) and $\omega=2 \pi / T$ is the angular frequency (in radians per second), in which $\lambda$ is the wavelength (in meters) and $T$ is the period (in seconds). According to (15), in formula (30) we set

$$
h_{0}=\text { const. }, \quad u_{0}=\frac{\Gamma}{2 \pi h_{0}} \ln (1+\varepsilon) .
$$

Substituting the presentation (30) into the first shallow water model (24)-(25) and keeping only the terms up to $0\left(\varepsilon^{2}\right)$, we obtain the following dispersion relation written in terms of the phase speed:

$$
C_{F_{1}}=\frac{\omega_{F_{1}}}{k}=\frac{A_{1} \pm \sqrt{B_{1}}}{C_{1}}
$$

where

$$
\begin{gathered}
A_{1}=\left[4 u_{0} h_{0}^{2}\left(k^{2}+9\right)+6 h_{0}\right] \varepsilon^{2}+12 u_{0}\left(1+3 \varepsilon h_{0}\right), \\
B_{1}=\left[h_{0}^{4} u_{0}^{2}\left(-414-60 k^{2}\right)+h_{0}^{4}\left(48 k^{3}+684 k\right)+36 h_{0}^{2}-252 h_{0}^{3} u_{0}\right] \varepsilon^{4}+ \\
{\left[h_{0}^{4}\left(684+48 k^{2}\right)+h_{0}^{3}\left(504 k-378 u_{0}^{2}\right)-72 h_{0}^{2} u_{0}\right] \varepsilon^{3}+} \\
{\left[h_{0}^{3}\left(1188+48 k^{2}\right)+h_{0}^{2}\left(144 k-144 u_{0}^{2}\right)\right] \varepsilon^{2}+648 h_{0}^{2} \varepsilon+144 h_{0},}
\end{gathered}
$$




$$
C_{1}=\varepsilon^{2} h_{0}^{2}\left(57+4 k^{2}\right)+42 h_{0} \varepsilon+12 .
$$

Correspondingly, substituting the presentation (30) into the second shallow water model (26)(27) and keeping only the terms up to $0\left(\varepsilon^{2}\right)$, we obtain the following dispersion relation:

$$
C_{F_{2}}=\frac{\omega_{F_{2}}}{k}=\frac{A_{2} \pm \sqrt{B_{2}}}{C_{2}}
$$

where

$$
\begin{gathered}
A_{2}=2 u_{0} h_{0}^{2}\left(2 k^{2}+3\right) \varepsilon^{2}+12 u_{0}\left(1+\varepsilon h_{0}\right), \\
B_{2}=\left[h_{0}^{4} u_{0}^{2}\left(45+24 k^{2}\right)+h_{0}^{5}\left(108+48 k^{2}\right)\right] \varepsilon^{4}+ \\
{\left[h_{0}^{4}\left(108-48 k^{2}\right)-72 h_{0}^{3} u_{0}^{2}\right] \varepsilon^{3}+} \\
{\left[-36 h_{0}^{2} u_{0}^{2}+h_{0}^{3}\left(36+48 k^{2}\right)\right] \varepsilon^{2}+72 h_{0}^{2} \varepsilon+144 h_{0},} \\
C_{2}=\varepsilon^{2} h_{0}^{2}\left(9+4 k^{2}\right)+18 h_{0} \varepsilon+12 .
\end{gathered}
$$

The positive and negative sing in the dispersion relations (32) and (36) correspond to the waves travelling in the positive or negative $\theta$-direction.

In order to illustrate the splitting phenomenon for the non-stationary case, we plot the phase speed $C_{F_{1}}=\omega_{F_{1}} / k$ and $C_{F_{2}}=\omega_{F_{2}} / k$ versus the wave number $k$ for the fixed values of parameter $\varepsilon$ that can be associated with the characteristics of the Earth: $R=6.384 \times 10^{3} \mathrm{~m}$.

As we can see from the plot shown in Figures $5-7$, showing the phase speeds $c_{F_{1}}$ and $c_{F_{2}}$ with different values of $\varepsilon$ associated with the models (24)-(25) and (26)-(27) versus the wave number $k$, the significant difference between two shallow water models becomes significant for smaller values of $k$ (long waves). However, the dispersion curves converge for any value of $h_{0}$ in the limit when $k \rightarrow \infty$ (short waves). The convergence takes place for smaller values of $k$ for smaller values of $h_{0}$. For example, the value $h_{0}=200 \mathrm{~km}$ can be associated with the mesosphere and the value $h_{0}=20 \mathrm{~km}$ can be associated with the troposphere, as shown schematically in Figure 4. However, we observe that the phase speed $c_{F_{i}}(i=1,2)$ is independent of the wavelength in the model in which we keep only $0\left(\varepsilon^{2}\right)$ terms so that the shallow water waves, at least up to the order $\varepsilon$, do not have frequency dispersion. Namely, in the shallow water models (24)-(25) and $(26)-(27)$, in which we keep only up to $0(\varepsilon)$ terms, we have:

$$
\begin{gathered}
a_{1}=2 u_{0}+\varepsilon u_{0} h_{0}, \\
b_{1}=\left(4 h_{0}^{2} u_{0}^{2}+6 h_{0}^{3}\right) \varepsilon^{2}-2 h_{0}^{2} \varepsilon-4 h_{0} \\
c_{1}=3 h_{0} \varepsilon+2
\end{gathered}
$$

Similarly,

$$
C_{F_{2}}=\frac{\omega_{F_{2}}}{k}=\frac{a_{2} \pm \sqrt{b_{2}}}{c_{2}}
$$

where

$$
\begin{gathered}
a_{2}=2 u_{0}+5 \varepsilon u_{0} h_{0}, \\
b_{2}=\left(4 h_{0}^{2} u_{0}^{2}-14 h_{0}^{3}\right) \varepsilon^{2}-18 h_{0}^{2} \varepsilon-4 h_{0} \\
c_{2}=7 h_{0} \varepsilon+2 .
\end{gathered}
$$




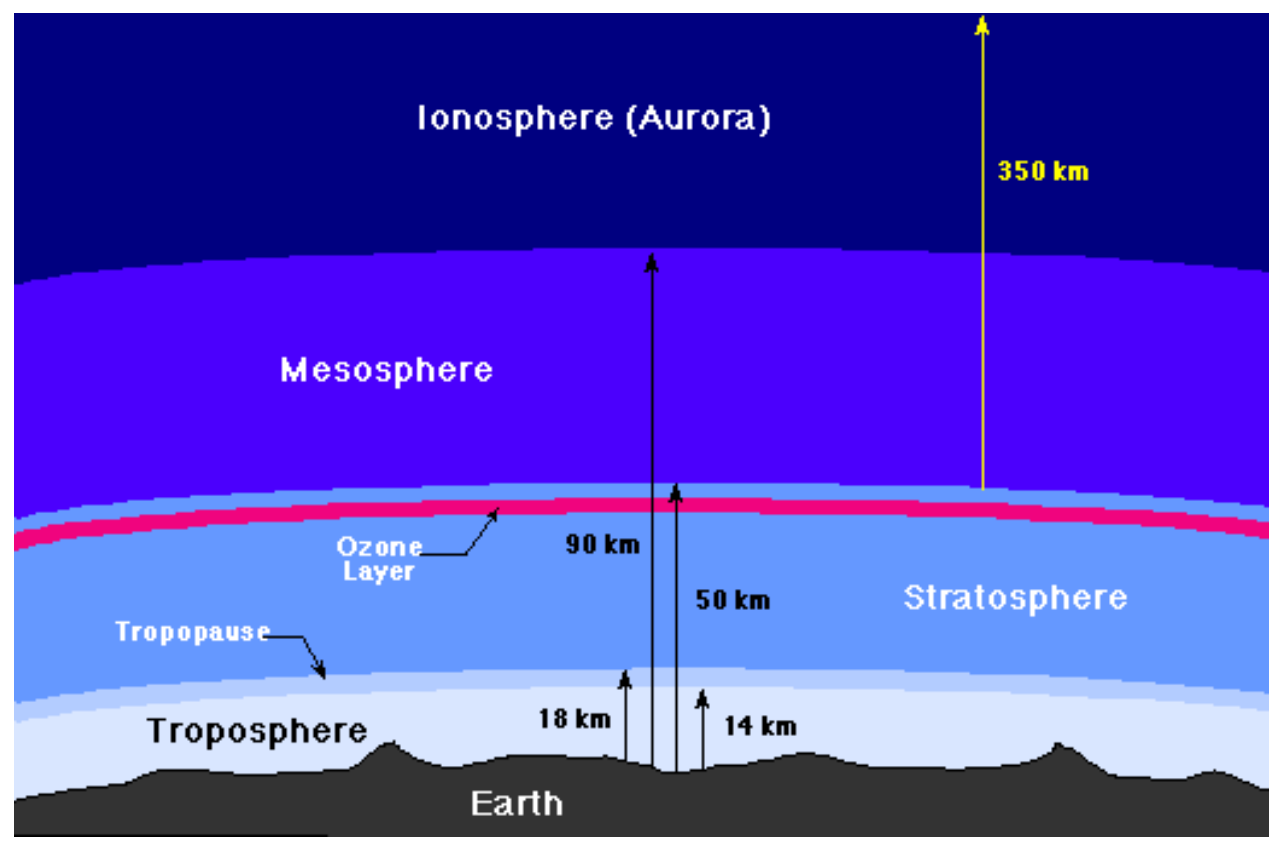

Figure 4: Schematic showing the atmospheric stratification. The troposphere, by definition, is the lowest 15-20 $\mathrm{km}$ layer of the atmosphere in which very sharp temperature decrease (from $-60^{0} \mathrm{C}$ to $+15^{0} \mathrm{C}$ ) is observed. The stratosphere is the higher 40-50 km layer of the atmosphere in which the temperature no longer decreases as it does in the troposphere, but instead becomes constant or increases slightly with height (from $-60^{0} \mathrm{C}$ to $\left.0^{0} C\right)$.

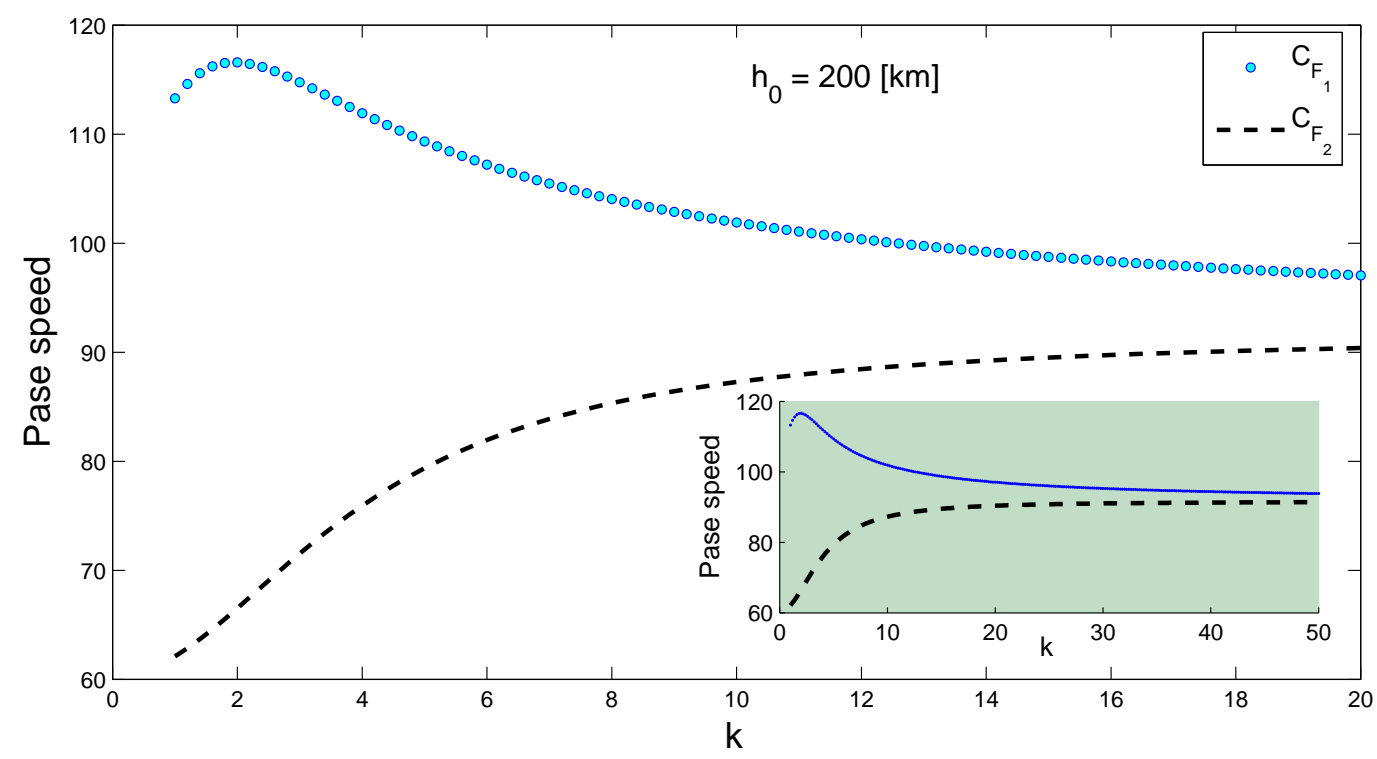

Figure 5: Comparison of the dispersion curves for the both shallow water models (24) - (25) when $h_{0}=200$ $\mathrm{km}$. 


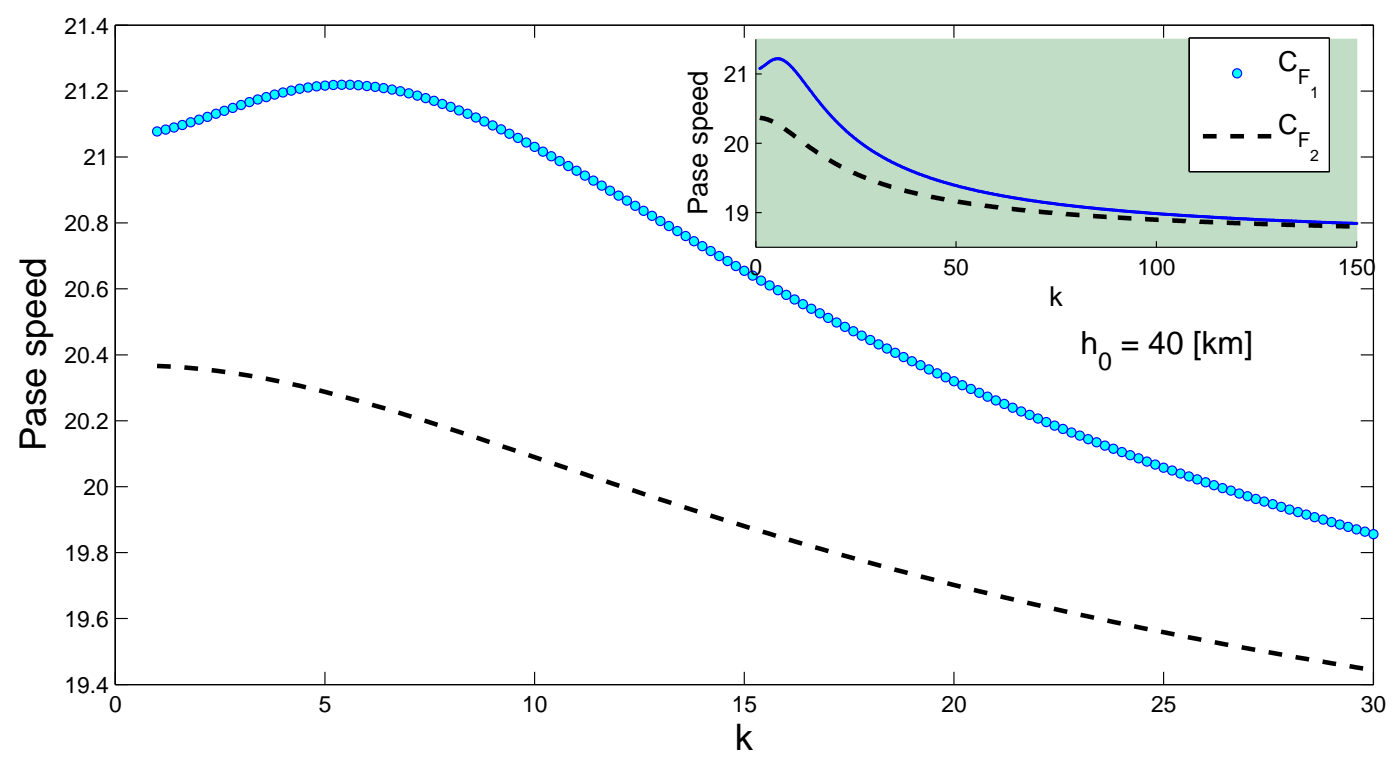

Figure 6: Comparison of the dispersion curves for the both shallow water models (24) - (25) when $h_{0}=40$ $\mathrm{km}$.

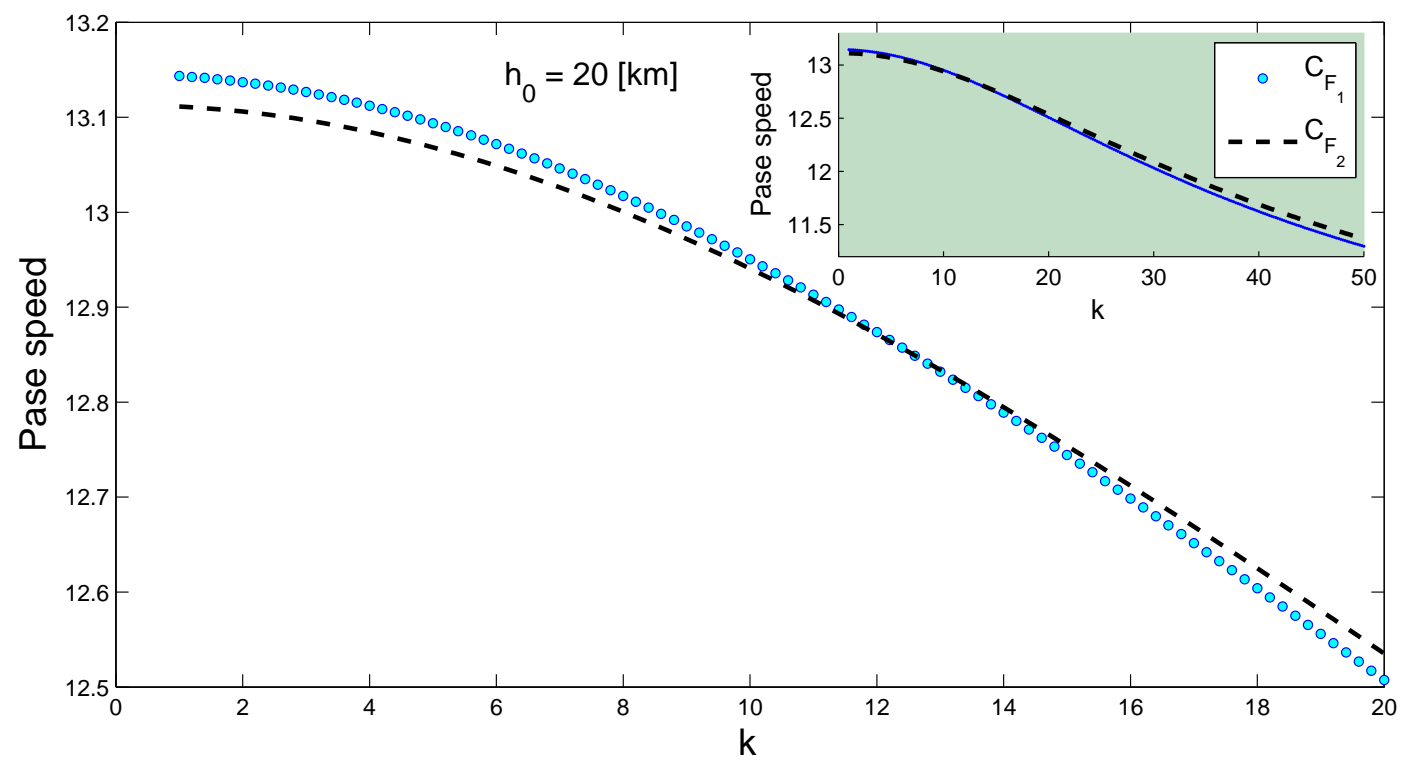

Figure 7: Comparison of the dispersion curves for the both shallow water models (24) - (25) when $h_{0}=20$ $\mathrm{km}$. 


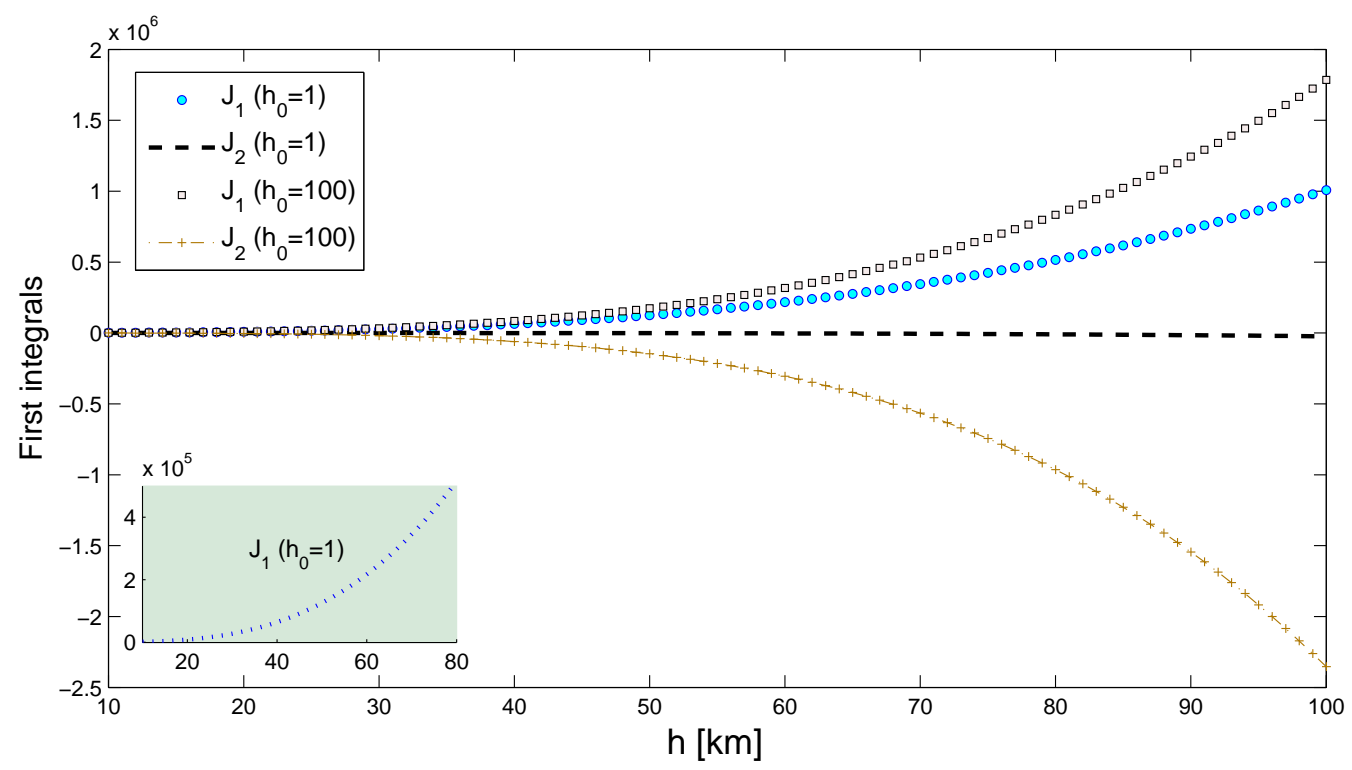

Figure 8: Comparison of the first integrals $J_{1}$ and $J_{2}$ with the choice of parameters $h_{0}=1$ and $h_{0}=100$.

\section{Concluding remarks}

In summary, we have shown that the higher order approximation of shallow water theory corresponding to the Cauchy-Poisson free boundary problem associated with a nonstationary motion of a perfect incompressible fluid circulating around the equatorial plane of a planet splits into two functionally independent systems. This situation was never observed for the classical flat-boundary problem. In order to illustrate the splitting phenomena, we have considered the linear non-stationary dispersion analysis. However, this situation can also be illustrated in the non-linear stationary case.

As an illustrative example let us consider the both systems models (24)-(25) and (26)-(27) in the stationary case up the order $0(\varepsilon)$. One can show that, in this case, the system (24)-(25) admits the following first integral:

$$
J_{1}=\frac{c_{1}^{2}}{2 h^{2}}+h+\varepsilon\left(\frac{5}{2} \frac{c_{1}^{2}}{2 h}+\frac{h}{2}\right)
$$

where $c_{1}$ and $J_{1}$ are constants of integrating. Similarly, the system (26)-(27) admits the first integral

$$
J_{2}=\frac{c_{1}^{2}}{2 h^{2}}+h+\varepsilon\left(\frac{5}{2} \frac{c_{1}^{2}}{2 h}+\frac{h}{2}-2 h\left[\frac{c_{1}^{2}}{2 h^{2}}+h\right]\right)
$$

where, again, $J_{2}$ is a constant of integrating.

This situation is illustrated in Figure 8, in which we plot the splitting of the corresponding values of $J_{1}$ and $J_{2}$ versus $h$ for different values of $h_{0}$.

The interest to this work has been motivated by the increasing interest of oceanographic community to mathematical modeling of atmospheric equatorial motion. Overall, equatorial 
planetary waves play a key role in atmospheric mixing processes, especially in stratospheretroposphere exchange, southern hemisphere climate response to ozone changes and in atmospheric variability ([10]; [12]; [13]; [14]; [37]). Understanding of the dynamics of such planetary waves plays a vital role in studying vertically propagating coastal waves at law latitudes and variability of the tropical oceans ([33]; [34]; [35]; [36]).

Acknowledgments: This research was supported in part by an appointment to the U.S. Department of Energy's Visiting Faculty Program.

\section{References}

[1] Anderson, R.F., Ali, S., Brandtmiller, L.L., Nielsen, S.H., Fleisher, M.Q. 2009: Winddriven upwelling in the Southern Ocean and the deglacial rise in atmospheric $\mathrm{CO}_{2}$, Science 323, 1443 .

[2] Astronomy, March 2013.

[3] Bachelor, G.K., 1967: An Introduction to Fluid Dynamics, Cambridge University Press, Cambridge .

[4] Bell, J. et al. 2001: Hubble captures best view of mars ever obtained From Earth. HubbleSite. NASA. http://hubblesite.org/newscenter/archive/releases/2001/24. Retrieved 201002-2\%.

[5] Ben-Yu, G., 1995: Spectral method for vorticity equations on spherical surface, Math. Comput. 64, 1067 .

[6] Blinova, E.N., 1943: A hydrodynamical theory of pressure and temperature waves and of centers of atmospheric action, C. R. Dokl. Acad. Sci.URSS 39, 257.

[7] Blinova, E.N., A method of solution of the nonlinear problem of atmospheric motions on a planetary scale, Dokl. Akad. Nauk SSSR N.S.110, 9751956.

[8] Boehm, M. T., Lee, S., 2003: The implications of tropical Rossby waves for tropical tropopause cirrus formation and for the equatorial upwelling of the Brewer-Dobson circulation. J. Atmos. Sci., 60, 247-261.

[9] Crapper, G. D. 1984: Introduction to Water Waves, Ellis Horwood, London.

[10] Edmon, H. J.,B. Hoskins, J., McIntyre, M.E., 1980: Eliassen-Palm cross sections for troposphere. J. Atmos. Sci., 37, 2600-2616.

[11] Friedrichs, K.O., Hyers, D.H., 1954: Thwe existence of solitary waves, Comm. Pure. Appl. Math. $\%$.

[12] Grise, K.M., Thompso, D. W. 2012: Equatorial Planetary Waves and Their Signature in Atmospheric Variability, J. Atmos. Sci., 69 (3), 857-874.

[13] Herrmann, E., 1896: The motions of the atmosphere and especially its waves, Bull. Am. Math. Soc. 2, 285. 
[14] Holton, J. R., Haynes, P.H., McIntyre, M.E. Douglass, A.R., Rood, C.D., Pfister, L., 1995: Stratosphere-troposphere exchange. Rev. Geophys., 33, 403-439.

[15] Ibragimov, N.H., Ibragimov, R.N., 2011: Integration by quadratures of the nonlinear Euler quations modeling atmospheric flows in a thin rotating spherical shell, Phys. Lett. A., 375, 3858-3865.

[16] Ibragimov, R.N., 2011: Nonlinear viscous fluid patterns in a thin rotating spherical domain and applications, Phys. Fluids, 23, 123102.

[17] Ibragimov, R.N, Jefferson, G., Carminati, J., 2013: Invariant and approximately invariant solutions of non-linear internal gravity waves forming a column of stratified fluid affected by the Earth's rotation, Int. J. Non-Linear Mech., 51, 28-44.

[18] Ibragimov, R.N, Jefferson, G., Carminati, J., 2013: Explicit invariant solutions associated with nonlinear atmospheric fllows in a thin rotating spherical shell with and without westto-east jets perturbations, Springer: anal. Math. Phys., 3 (3), 201-294.

[19] Ibragimov, R.N., Pelinovsky, D.E., 2009: Incompressible viscous fluid flows in a thin spherical shell, J. Math. Fluid Mech. 11, 60.

[20] Ibragimov, R.N., 2001: On the tidal motion around the earth complicated by the circular geometry of the ocean's shape without Coriolis forces, Math Phys, Analysis and Geom., 4, 51-63.

[21] Ibragimov, R.N., Villasenor, H., 2014: Energy Balance Associated With a Mixing Process at the Interface of a Two-Layer Longitudinal Atmospheric Model, J. Fluids Eng. 136(7), doi:10.1115/1.4026857

[22] Ibragimov, R.N., Ibragimov, N.H., Galiakberova, L.R., 2014: Symmetries and conservation laws of a spectral nonlinear model for atmospheric baroclinic jets, Math. Model. Nat. Phenom.9 (5), 32-39.

[23] Ibragimov, R.N., Aitbayev, R., 2009: Three-dimensional non-linear rotating surface waves in channels of variable depth in the presence of formation of a small perturbation of atmospheric pressure across the channel, Commun. Nonlinear Sci. Numer. Simulat. 14, 3811-3820.

[24] Ibragimov, R.N., 2010: Free boundary effects on stability of two phase planar fluid motion in annulus: Migration of the stable mode. Comm. Nonlinear Sci. Numer. Simulat. 15 (9), 2361-2374.

[25] Ibragimov, R.N., Dameron, M., Dannangoda, C., 2012: Sources and sinks of energy balance for nonlinear atmospheric motion perturbed by west-to-east winds progressing on a surface of rotating spherical shell, Discontinuity, Nonlinearity and Complexity, 1 (1), 41-55.

[26] Ibragimov, R.N., Pelinovsky, D., 2007: Three-dimensional gravity waves in a channel of variable depth, Commun. Nonlinear Sci. Numer. Simulat. 13, 2104-2133. 
[27] Iftimie, D., Raugel, G., 2001: Some results on the NS equations in thin three-dimensional domains, J. Differ. Equations 169, 281.

[28] Lions, L., Teman, R., Wang, S., 1992: On the equations of the large-scale ocean, Nonlinearity 5, 1007 .

[29] Lions, L., Teman, R., Wang, S., 1992: New formulations of the primitive equations of atmosphere and applications, Nonlinearity 5, 237.

[30] Okamoto, H., 1986: Nonstationary free boundary problem for perfect fluid with surface tension, J. Math. Soc. Japan 38 (3).

[31] Saber, R., 2012: 2012 A Family Brief: "the Science Is All In", Xlibris Corporation, 2009.

[32] Stoker, J.J., 1957: Water waves, Interscience Publishers, Inc., New York.

[33] Toggweiler, R., Russel, J.L., 2008: Ocean circulation on a warming climate, Nature London 451, 286 .

[34] Philanders, S.G., 1978: Variability of the tropical oceans, Dynamics Oceans 8 Atmos. 3, 191.

[35] Romea, R.D., Allen, J.S., 1983: On vertically propagating coastal Kelvin waves at low latitudes. J. Phys. Oceanogr. 13 (1),241-1,254.

[36] Simmons, A. J., Hoskins, B.J., 1978: The life cycles of some nonlinear baroclinic waves. J. Atmos. Sci., 35, 414-432.

[37] Shindell, D.T., Schmidt, G.A., 2004: Southern hemisphere climate response to ozone changes and greenhouse gas increases, Geophys. Res. Lett. 31, L18209, doi:10.1029/2004GL020724.

[38] Stokes, G. G. 1847: On the theory of oscillatory waves, Trans. Cambridge Philos. Soc. 8, $441-455$.

[39] Summerhayes, C.P., Thorpe, S.A., 1996: Oceanography: An Illustrative Guide, Wiley, New York .

[40] Swarztrauber, P.N., 2004: Shallow water flow on the sphere, Mon. Weather Rev. 132, 3010

[41] Weijer, W., Vivier, F., Gille, S.T., Dijkstra, H., 2007: Multiple oscillatory modes of the Argentine Basin. Part II: The spectral origin of basin modes, J. Phys. Oceanogr. 37, 2869 